\title{
A Note on Minimal Hypersurfaces of an Odd Dimensional Sphere
}

\author{
Sharief Deshmukh ${ }^{1, *}$ and Ibrahim Al-Dayel ${ }^{2}$ (D) \\ 1 Department of Mathematics, College of Science, King Saud University, P.O.Box-2455, Riyadh 11451, \\ Saudi Arabia \\ 2 Department of Mathematics and Statistics, College of Science, Imam Muhammad Ibn Saud Islamic \\ University, P.O. Box-65892, Riyadh 11566, Saudi Arabia; iaaldayel@imamu.edu.sa \\ * Correspondence: shariefd@ksu.edu.sa
}

Received: 17 January 2020; Accepted: 11 February 2020; Published: 21 February 2020

\begin{abstract}
We obtain the Wang-type integral inequalities for compact minimal hypersurfaces in the unit sphere $S^{2 n+1}$ with Sasakian structure and use these inequalities to find two characterizations of minimal Clifford hypersurfaces in the unit sphere $S^{2 n+1}$.
\end{abstract}

Keywords: clifford minimal hypersurfaces; sasakian structure; integral inequalities; reeb function; contact vector field

MSC: 53C40; 53C42; 53C25

\section{Introduction}

Let $M$ be a compact minimal hypersurface of the unit sphere $S^{n+1}$ with shape operator $A$. In his pioneering work, Simons [1] has shown that on a compact minimal hypersurface $M$ of the unit sphere $S^{n+1}$ either $A=0$ (totally geodesic), or $\|A\|^{2}=n$, or $\|A\|^{2}(p)>n$ for some point $p \in M$, where $\|A\|$ is the length of the shape operator. This work was further extended in [2] and for compact constant mean curvature hypersurfaces in [3]. If for every point $p$ in $M$, the square of the length of the second fundamental form of $M$ is $n$, then it is known that $M$ must be a subset of a Clifford minimal hypersurface

$$
S^{l}\left(\sqrt{\frac{l}{n}}\right) \times S^{m}\left(\sqrt{\frac{m}{n}}\right)
$$

where $l, m$ are positive integers, $l+m=n$ (cf. Theorem 3 in [4]). Note that this result was independently proven by Lawson [2] and Chern, do Carmo, and Kobayashi [5]. One of the interesting questions in differential geometry of minimal hypersurfaces of the unit sphere $S^{n+1}$ is to characterize minimal Clifford hypersurfaces. Minimal hypersurfaces have also been studied in (cf. [6-8]). In [2], bounds on Ricci curvature are used to find a characterization of the minimal Clifford hypersurfaces in the unit sphere $S^{4}$. Similarly in [3,9-11], the authors have characterized minimal Clifford hypersurfaces in the odd-dimensional unit spheres $S^{3}$ and $S^{5}$ using constant contact angle. Wang [12] studied compact minimal hypersurfaces in the unit sphere $S^{n+1}$ with two distinct principal curvatures, one of them being simple and obtained the following integral inequality,

$$
\int_{M}\|A\|^{2} \leq n \operatorname{Vol}(M)
$$


where $\operatorname{Vol}(M)$ is the volume of $M$. Moreover, he proved that equality in the above inequality holds if and only if $M$ is the Clifford hypersurface,

$$
S^{1}\left(\sqrt{\frac{1}{n}}\right) \times S^{m}\left(\sqrt{\frac{n-1}{n}}\right) .
$$

In this paper, we are interested in studying compact minimal hypersurfaces of the unit sphere $S^{2 n+1}$ using the Sasakian structure $(\varphi, \xi, \eta, g)$ (cf. [13]) and finding characterizations of minimal Clifford hypersurface of $S^{2 n+1}$. On a compact minimal hypersurface $M$ of the unit sphere $S^{2 n+1}$, we denote by $N$ the unit normal vector field and define a smooth function $f=g(\xi, N)$, which we call the Reeb function of the minimal hypersurface $M$. Also, on the hypersurface $M$, we have a smooth vector field $v=\varphi(N)$, which we call the contact vector field of the hypersurface ( $v$ being orthogonal to $\xi$ belongs to contact distribution). Instead of demanding two distinct principal curvatures one being simple, we ask the contact vector field $v$ of the minimal hypersurface in $S^{2 n+1}$ to be conformal vector field and obtain an inequality similar to Wang's inequality and show that the equality holds if and only if $M$ is isometric to a Clifford hypersurface. Indeed we prove

Theorem 1. Let $M$ be a compact minimal hypersurface of the unit sphere $S^{2 n+1}$ with Reeb function $f$ and contact vector field $v$ a conformal vector field on $M$. Then,

$$
\int_{M}\left(1-f^{2}\right)\|A\|^{2} \leq 2 n \int_{M}\left(1-f^{2}\right)
$$

and the equality holds if and only if $M$ is isometric to the Clifford hypersurface $S^{l}\left(\sqrt{\frac{l}{2 n}}\right) \times S^{m}\left(\sqrt{\frac{m}{2 n}}\right)$, where $l+m=2 n$.

Also in [12], Wang studied embedded compact minimal non-totally geodesic hypersurfaces in $S^{n+1}$ those are symmetric with respect to $n+2$ pair-wise orthogonal hyperplanes of $R^{n+2}$. If $M$ is such a hypersurface, then it is proved that

$$
\int_{M}\|A\|^{2} \geq n \operatorname{Vol}(M)
$$

and the equality holds precisely if $M$ is a Clifford hypersurface. Note that compact embedded hypersurface has huge advantage over the compact immersed hypersurface, as it divides the ambient unit sphere $S^{n}$ into two connected components.

In our next result, we consider compact immersed minimal hypersurface $M$ of the unit sphere $S^{2 n+1}$ such that the Reeb function $f$ is a constant along the integral curves of the contact vector field $v$ and show that above inequality of Wang holds, and we get another characterization of minimal Clifford hypersurface in the unit sphere $S^{2 n+1}$. Precisely, we prove the following.

Theorem 2. Let $M$ be a compact minimal hypersurface of the unit sphere $S^{2 n+1}$ with Reeb function $f$ a constant along the integral curves of the contact vector field $v$. Then,

$$
\int_{M}\|A\|^{2} \geq 2 n \operatorname{Vol}(M)
$$

and the equality holds if and only if $M$ is isometric to the Clifford hypersurface $S^{l}\left(\sqrt{\frac{l}{2 n}}\right) \times S^{m}\left(\sqrt{\frac{m}{2 n}}\right)$, where $l+m=2 n$. 


\section{Preliminaries}

Recall that conformal vector fields play an important role in the geometry of a Riemannian manifolds. A conformal vector field $v$ on a Riemannian manifold $(M, g)$ has local flow consisting of conformal transformations, which is equivalent to

$$
£_{v} g=2 \rho g
$$

The smooth function $\rho$ appearing in Equation (1) defined on $M$ is called the potential function of the conformal vector field $v$. We denote by $(\varphi, \xi, \eta, g)$ the Sasakian structure on the unit sphere $S^{2 n+1}$ as a totally umbilical real hypersurface of the complex space form $\left(C^{n+1}, \bar{J},\langle\rangle,\right)$, where $\bar{J}$ is the complex structure and $\langle$,$\rangle is the Euclidean Hermitian metric. The Sasakian structure (\varphi, \xi, \eta, g)$ on $S^{2 n+1}$ consists of a $(1,1)$ skew symmetric tensor field $\varphi$, a smooth unit vector field $\xi$, a smooth 1 -form $\eta$ dual to $\xi$, and the induced metric $g$ on $S^{2 n+1}$ as real hypersurface of $C^{n+1}$ and they satisfy (cf. [13])

$$
\varphi^{2}=-I+\eta \otimes \xi, \eta \circ \varphi=0, \eta(\xi)=1, g(\varphi X, \varphi Y)=g(X, Y)-\eta(X) \eta(Y),
$$

and

$$
(\bar{\nabla} \varphi)(X, Y)=g(X, Y) \xi-\eta(Y) X, \quad \bar{\nabla}_{X} \xi=-\varphi X
$$

where $X, Y$ are smooth vector fields, $\bar{\nabla}$ is Riemannian connection on $S^{2 n+1}$ and the covariant derivative

$$
(\bar{\nabla} \varphi)(X, Y)=\bar{\nabla}_{X} \varphi Y-\varphi\left(\bar{\nabla}_{X} Y\right)
$$

We dente by $N$ and $A$ the unit normal and the shape operator of the hypersurface $M$ of the unit sphere $S^{2 n+1}$. We denote the induced metric on the hypersurface $M$ by the same letter $g$ and denote by $\nabla$ the Riemannian connection on the hypersurface $M$ with respect to the induced metric $g$. Then, the fundamental equations of hypersurface are given by (cf. [14])

$$
\begin{gathered}
\bar{\nabla}_{X} Y=\nabla_{X} Y+g(A X, Y), \bar{\nabla}_{X} N=-A X, \quad X, Y \in \mathfrak{X}(M), \\
R(X, Y) Z=g(Y, Z) X-g(X, Z) Y+g(A Y, Z) A X-g(A X, Z) A Y, \\
(\nabla A)(X, Y)=(\nabla A)(Y, X), \quad X, Y \in \mathfrak{X}(M),
\end{gathered}
$$

where $\mathfrak{X}(M)$ is the Lie algebra of smooth vector fields and $R(X, Y) Z$ is the curvature tensor field of the hypersurface $M$. The Ricci tensor of the minimal hypersurface $M$ of the unit sphere $S^{2 n+1}$ is given by

$$
\operatorname{Ric}(X, Y)=(2 n-1) g(X, Y)-g(A X, A Y), \quad X, Y \in \mathfrak{X}(M)
$$

and

$$
\sum_{i=1}^{2 n}(\nabla A)\left(e_{i}, e_{i}\right)=0
$$

holds for a local orthonormal frame $\left\{e_{1}, \ldots, e_{2 n}\right\}$ on the minimal hypersurface $M$.

Using the Sasakian structure $(\varphi, \xi, \eta, g)$ on the unit sphere $S^{2 n+1}$, we analyze the induced structure on a hypersurface $M$ of $S^{2 n+1}$. First, we have a smooth function $f$ on the hypersurface $M$ defined by $f=g(\xi, N)$, which we call the Reeb function of the hypersurface $M$, where $N$ is the unit normal vector field. As the operator $\varphi$ is skew symmetric, we get a vector field $v=\varphi N$ defined on $M$, which we call the contact vector field of the hypersurface $M$. Note that the vector field $v$ is orthogonal to $\xi$, and therefore lies in the contact distribution of the Sasakian manifold $S^{2 n+1}$. We denote by $u=\xi^{T}$ the tangential component of $\xi$ to the hypersurface $M$ and, consequently, we have $\xi=u+f N$. Let $\alpha$ and $\beta$ be smooth 1 -forms on $M$ dual to the vector fields $u$ and $v$, respectively, that is, $\alpha(X)=g(X, u)$ and $\beta(X)=g(X, v), X \in \mathfrak{X}(M)$. For $X \in \mathfrak{X}(M)$, we set $J X=(\varphi X)^{T}$ the tangential component of $\varphi X$ to the hypersurface, which gives a skew symmetric $(1,1)$ tensor field $J$ on the hypersurface $M$. It follows 
that $\varphi X=J X-\beta(X) N$. Thus, we get a structure $(J, u, v, \alpha, \beta, f, g)$ on the hypersurface $M$ and using properties in Equations (2) and (3) of the Sasakian structure $(\varphi, \xi, \eta, g)$ on the unit sphere $S^{2 n+1}$ and Equation (4), it is straightforward to see that the structure $(J, u, v, \alpha, \beta, f, g)$ on the hypersurface $M$ has the properties described in the following Lemma.

Lemma 1. Let $M$ be a hypersurface of the unit sphere $S^{2 n+1}$. Then, $M$ admits the structure $(J, u, v, \alpha, \beta, f, g)$ satisfying

(i) $J^{2}=-I+\alpha \otimes u+\beta \otimes v$,

(ii) $J u=-f v, \quad J v=f u$,

(iii) $g(J X, J Y)=g(X, Y)-\alpha(X) \alpha(Y)-\beta(X) \beta(Y)$,

(iv) $\nabla_{X} u=-J X+f A X, \quad \nabla_{X} v=-f X-J A X$,

(v) $(\nabla J)(X, Y)=g(X, Y) u-\alpha(Y) X+g(A X, Y) v-\beta(Y) A X$,

(vi) $\nabla f=-A u+v$,

(vii) $\|u\|^{2}=\|v\|^{2}=\left(1-f^{2}\right), \quad g(u, v)=0$,

where $\nabla f$ is the gradient of the Reeb function $f$.

Let $\Delta f$ be the Laplacian of the Reeb function $f$ of the minimal hypersurface $M$ of the unit sphere $S^{2 n+1}$ defined by $\Delta f=\operatorname{div} \nabla f$. Then using Lemma 1 and $\frac{1}{2} \Delta f^{2}=f \Delta f+\|\nabla f\|^{2}$ and Equations (6) and (8), we get the following:

Lemma 2. Let $M$ be a minimal hypersurface of the unit sphere $S^{2 n+1}$. Then, the Reeb function $f$ satisfies

$$
\begin{aligned}
& \Delta f=-\left(2 n+\|A\|^{2}\right) f, \\
& \text { (ii) } \frac{1}{2} \Delta f^{2}=-\left(2 n+\|A\|^{2}\right) f^{2}+\|\nabla f\|^{2} \text {. }
\end{aligned}
$$

On the hypersurface $M$ of the unit sphere $S^{2 n+1}$, we define a $(1,1)$ tensor field $\Psi=J A-A J$, then it follows that $g(\Psi X, Y)=g(X, \Psi Y), X, Y \in \mathfrak{X}(M)$, that is, $\Psi$ is symmetric and that $\operatorname{tr} \Psi=0$. Next, we prove the following:

Lemma 3. Let $M$ be a compact minimal hypersurface of the unit sphere $S^{2 n+1}$. Then,

$$
\int_{M}\left(1-f^{2}\right)\|A\|^{2}=\int_{M}\left(2 n-2 n(2 n+1) f^{2}+\frac{1}{2}\|\Psi\|^{2}\right)
$$

Proof. Using Equation (7), we have $\operatorname{Ric}(v, v)=(2 n-1)\|v\|^{2}-\|A v\|^{2}$. Now, using Lemma 1, we get

$$
\left(£_{v} g\right)(X, Y)=-2 f g(X, Y)-g(\Psi X, Y)
$$

which on using the fact that $t r \Psi=0$, gives

$$
\left|£_{v} g\right|^{2}=8 n f^{2}+\|\Psi\|^{2} .
$$

Also, using (iii) of Lemma 1, we have

$$
\|J A\|^{2}=\|A\|^{2}-\|A u\|^{2}-\|A v\|^{2},
$$

which together with second equation in (iv) of Lemma 1 and the fact that $\operatorname{tr} J A=0$, implies

$$
\|\nabla v\|^{2}=2 n f^{2}+\|A\|^{2}-\|A u\|^{2}-\|A v\|^{2} .
$$


Note that second equation in (iv) of Lemma 1 also gives

$$
\operatorname{div} v=-2 n f .
$$

Now, inserting above values in the following Yano's integral formula (cf. [15])

$$
\int_{M}\left(\operatorname{Ric}(v, v)+\frac{1}{2}\left|\hat{E}_{v} g\right|^{2}-\|\nabla v\|^{2}-(\operatorname{div} v)^{2}\right)=0,
$$

we get

$$
\int_{M}\left((2 n-1)\|v\|^{2}+2 n f^{2}+\frac{1}{2}\|\Psi\|^{2}-\|A\|^{2}+\|A u\|^{2}-4 n^{2} f^{2}\right)=0 .
$$

Also, (vi) of Lemma 1, gives $A u=v-\nabla f$, that is, $\|A u\|^{2}=\|v\|^{2}+\|\nabla f\|^{2}-2 v(f)$, which on using $\operatorname{div}(f v)=v(f)+f \operatorname{div} v=v(f)-2 n f^{2}$, gives

$$
\|A u\|^{2}=\|v\|^{2}+\|\nabla f\|^{2}-2 \operatorname{div}(f v)-4 n f^{2} .
$$

Inserting above value of $\|A u\|^{2}$ in Equation (9), yields

$$
\int_{M}\left(2 n\|v\|^{2}-2 n f^{2}+\frac{1}{2}\|\Psi\|^{2}-\|A\|^{2}+\|\nabla f\|^{2}-4 n^{2} f^{2}\right)=0 .
$$

Integrating (ii) of Lemma 2, we get

$$
\int_{M}\|\nabla f\|^{2}=\int_{M}\left(2 n+\|A\|^{2}\right) f^{2}
$$

which together with $\|v\|^{2}=1-f^{2}$ and Equation (10) proves the integral formula.

Lemma 4. Let $M$ be a minimal hypersurface of the unit sphere $S^{2 n+1}$. Then, the contact vector field $v$ is a conformal vector field if and only if $J A=A J$.

Proof. Suppose that $A J=J A$. Then, using Lemma 1 and symmetry of shape operator $A$ and skew symmetry of the operator $J$, we have

$$
\left(\ell_{v} g\right)(X, Y)=g\left(\nabla_{X} v, Y\right)+g\left(\nabla_{Y} v, X\right)=-2 f g(X, Y), \quad X \in \mathfrak{X}(M),
$$

which proves that $v$ is a conformal vector field with potential function $-f$. Conversely, suppose $v$ is conformal vector field with potential function $\rho$. Then, using Equation (1), we have

$$
\left(\ell_{v} g\right)(X, Y)=g\left(\nabla_{X} v, Y\right)+g\left(\nabla_{Y} v, X\right)=2 \rho g(X, Y) \text {, }
$$

which on using Lemma 1, gives

$$
g(-J A X-f X, Y)+g(-J A Y-f Y, X)=2 \rho g(X, Y)
$$

that is,

$$
g(A J X-J A X, Y)=2(\rho+f) g(X, Y) .
$$

Choosing a local orthonormal frame $\left\{e_{1}, \ldots, e_{2 n}\right\}$ on the minimal hypersurface $M$ and taking $X=Y=e_{i}$ in above equation and summing, we get $\rho=-f$. This gives $g(A J X-J A X, Y)=0$, $X, Y \in \mathfrak{X}(M)$, that is, $A J=J A$. 
Lemma 5. Let $M$ be a minimal hypersurface of the unit sphere $S^{2 n+1}$. If the contact vector field $v$ is a conformal vector field on $M$, then

$$
A u=\frac{\|A\|^{2}}{2 n} v .
$$

Proof. Suppose $v$ is a conformal vector field. Then, by Lemma 4 , we have $J A=A J$. Note that for the Hessian operator $A_{f}$ of the Reeb function $f$ using Lemma 1, we have

$$
A_{f}(X)=\nabla_{X} \nabla f=\nabla_{X}(v-A u)=-J A X-f X-\nabla_{X} A u, \quad X \in \mathfrak{X}(M),
$$

which on using (vi) of Lemma 1, gives

$$
A_{f}(X)=-f\left(X+A^{2} X\right)-(\nabla A)(X, u) .
$$

Taking covariant derivative in above equation gives

$$
\begin{aligned}
\left(\nabla A_{f}\right)(X, Y)= & -X(f)\left(\left(Y+A^{2} Y\right)-f\left(\nabla A^{2}\right)(X, Y)-\left(\nabla^{2} A\right)(X, Y, u)\right. \\
& +(\nabla A)(Y, J X)-f(\nabla A)(Y, A X),
\end{aligned}
$$

where we used (iv) of Lemma 1 . Now, on taking a local orthonormal frame $\left\{e_{1}, \ldots, e_{2 n}\right\}$ on the minimal hypersurface $M$ and taking $X=Y=e_{i}$ in above equation and summing, we get

$$
\begin{aligned}
\sum_{i=1}^{2 n}\left(\nabla A_{f}\right)\left(e_{i}, e_{i}\right)= & -\nabla f-A^{2} \nabla f-f \sum_{i=1}^{2 n}\left(\nabla A^{2}\right)\left(e_{i}, e_{i}\right)-\sum_{i=1}^{2 n}\left(\nabla^{2} A\right)\left(e_{i}, e_{i}, u\right) \\
& +\sum_{i=1}^{2 n}(\nabla A)\left(e_{i}, J e_{i}\right)-f \sum_{i=1}^{2 n}(\nabla A)\left(e_{i}, A e_{i}\right) .
\end{aligned}
$$

Note that for the minimal hypersurface, we have

$$
\begin{aligned}
\sum_{i=1}^{2 n}(\nabla A)\left(e_{i}, A e_{i}\right) & =\sum_{i=1}^{2 n}\left(\nabla_{e_{i}} A^{2} e_{i}-A((\nabla A))\left(e_{i}, e_{i}\right)+A\left(\nabla_{e_{i}} e_{i}\right)\right) \\
& =\sum_{i=1}^{2 n}\left(\nabla A^{2}\right)\left(e_{i}, e_{i}\right) .
\end{aligned}
$$

Thus, the previous equation takes the form

$$
\sum_{i=1}^{2 n}\left(\nabla A_{f}\right)\left(e_{i}, e_{i}\right)=-\nabla f-A^{2} \nabla f-2 f \sum_{i=1}^{2 n}\left(\nabla A^{2}\right)\left(e_{i}, e_{i}\right)-\sum_{i=1}^{2 n}\left(\nabla^{2} A\right)\left(e_{i}, e_{i}, u\right)+\sum_{i=1}^{2 n}(\nabla A)\left(e_{i}, J e_{i}\right) .
$$

Now, using the definition of Hessian operator, we have

$$
R(X, Y) \nabla f=\left(\nabla A_{f}\right)(X, Y)-\left(\nabla A_{f}\right)(Y, X)
$$

which gives

$$
\operatorname{Ric}(Y, \nabla f)=g\left(Y, \sum_{i=1}^{2 n}\left(\nabla A_{f}\right)\left(e_{i}, e_{i}\right)\right)-Y(\Delta f)
$$

and we conclude

$$
Q(\nabla f)=-\nabla(\Delta f)+\sum_{i=1}^{2 n}\left(\nabla A_{f}\right)\left(e_{i}, e_{i}\right)
$$


where $Q$ is the Ricci operator defined by $\operatorname{Ric}(X, Y)=g(Q X, Y), X, Y \in \mathfrak{X}(M)$. Using (i) of Lemma 2, we have

$$
\nabla(\Delta f)=-2 n \nabla f-\|A\|^{2} \nabla f-f \nabla\|A\|^{2}
$$

and, consequently, using $Q(X)=(2 n-1) X-A^{2} X$ (outcome of Equation (7)), the Equation (12) takes the form

$$
\sum_{i=1}^{2 n}\left(\nabla A_{f}\right)\left(e_{i}, e_{i}\right)=(2 n-1) \nabla f-A^{2}(\nabla f)-2 n \nabla f-\|A\|^{2} \nabla f-f \nabla\|A\|^{2},
$$

that is,

$$
\sum_{i=1}^{2 n}\left(\nabla A_{f}\right)\left(e_{i}, e_{i}\right)=-\nabla f-A^{2}(\nabla f)-\|A\|^{2} \nabla f-f \nabla\|A\|^{2} .
$$

Also, note that

$$
\begin{aligned}
X\left(\|A\|^{2}\right) & =X\left(\sum_{i=1}^{2 n} g\left(A e_{i}, A e_{i}\right)\right)=2 \sum_{i=1}^{2 n} g\left((\nabla A)\left(X, e_{i}\right), A e_{i}\right) \\
& =2 \sum_{i=1}^{2 n} g\left(X,(\nabla A)\left(e_{i}, A e_{i}\right)\right),
\end{aligned}
$$

where we have used Equation (6) and symmetry of the shape operator $A$. Therefore, the gradient of the function $\|A\|^{2}$ is

$$
\nabla\|A\|^{2}=2 \sum_{i=1}^{2 n}(\nabla A)\left(e_{i}, A e_{i}\right),
$$

and, consequently, Equation (13), takes the form

$$
\sum_{i=1}^{2 n}\left(\nabla A_{f}\right)\left(e_{i}, e_{i}\right)=-\nabla f-A^{2}(\nabla f)-\|A\|^{2} \nabla f-2 f \sum_{i=1}^{2 n}(\nabla A)\left(e_{i}, A e_{i}\right) .
$$

Using Equations (11) and (14), we conclude

$$
-\|A\|^{2} \nabla f=-\sum_{i=1}^{2 n}\left(\nabla^{2} A\right)\left(e_{i}, e_{i}, u\right)+\sum_{i=1}^{2 n}(\nabla A)\left(e_{i}, J e_{i}\right) .
$$

Now, using Equations (6) and (8) and the Ricci identity, we have

$$
\sum_{i=1}^{2 n}\left(\nabla^{2} A\right)\left(e_{i}, e_{i}, u\right)=\sum_{i=1}^{2 n}\left(\nabla^{2} A\right)\left(e_{i}, u, e_{i}\right)=\sum_{i=1}^{2 n}\left(R\left(e_{i}, u\right) A e_{i}-A R\left(e_{i}, u\right) e_{i}\right),
$$

which on using Equation (5) and $\operatorname{tr} A=0$ gives

$$
\sum_{i=1}^{2 n}\left(\nabla^{2} A\right)\left(e_{i}, e_{i}, u\right)=-\|A\|^{2} A u+2 n A u .
$$

Also, using $J A=A J$, we have

$$
\begin{aligned}
\sum_{i=1}^{2 n}(\nabla A)\left(e_{i}, J e_{i}\right) & =\sum_{i=1}^{2 n}\left(\nabla_{e_{i}} J A e_{i}-A\left((\nabla J)\left(e_{i}, e_{i}\right)+J\left(\nabla_{e_{0}} e_{i}\right)\right)\right. \\
& =\sum_{i=1}^{2 n}\left((\nabla J)\left(e_{i}, A e_{i}\right)-A\left((\nabla J)\left(e_{i}, e_{i}\right)\right),\right.
\end{aligned}
$$


which on using (v) of Lemma 1, yields

$$
\sum_{i=1}^{2 n}(\nabla A)\left(e_{i}, J e_{i}\right)=\|A\|^{2} v-2 n A u .
$$

Finally, using (vi) of Lemma 1 and Equations (16) and (17) in Equation (15), we get

$$
-\|A\|^{2}(-A u+v)=\|A\|^{2} A u-2 n A u+\|A\|^{2} v-2 n A u
$$

and this proves the Lemma.

\section{Proof of Theorem 1}

As the contact vector field $v$ is a conformal vector field by Lemma 4 , we have $J A=A J$, that is, $\Psi=0$. Then Lemma 3 implies

$$
\int_{M}\left(1-f^{2}\right)\|A\|^{2}=\int_{M}\left(2 n-2 n(2 n+1) f^{2}\right),
$$

that is,

$$
\int_{M}\left(1-f^{2}\right)\|A\|^{2}=\int_{M}\left(2 n\left(1-f^{2}\right)-4 n f^{2}\right) .
$$

Therefore, we get the inequality

$$
\int_{M}\left(1-f^{2}\right)\|A\|^{2} \leq \int_{M} 2 n\left(1-f^{2}\right) .
$$

Moreover, if the equality holds, then by Equation (18), we get $f=0$, which in view of (vi), (vii) of Lemma 1 , we conclude that $A u=v$ and that the contact vector field $v$ is a unit vector field. As $v$ is a conformal vector field, combining $A u=v$ with Lemma 5, we get $\|A\|^{2} v=2 n v$, that is, $\|A\|^{2}=2 n$. Therefore, $M$ is a Clifford hypersurface (cf. [5]).

The converse is trivial.

\section{Proof of Theorem 2}

As the Reeb function $f$ is a constant along the integral curves of the contact vector field $v$, we have $v(f)=0$. Note that $\operatorname{div}(f v)=v(f)+f \operatorname{div} v=-2 n f^{2}$, which on integration gives $f=0$, and consequently, the contact vector field $v$ is a unit vector field. Then Lemma 3, implies

$$
\int_{M}\|A\|^{2}=\int_{M}\left(2 n+\frac{1}{2}\|\Psi\|^{2}\right)
$$

which proves the inequality

$$
\int_{M}\|A\|^{2} \geq 2 n \operatorname{Vol}(M)
$$

If the equality holds, then by Equation (4.1), we get that $\Psi=0$, that is, $J A=A J$. Thus, by Lemma 4 , the contact vector field $v$ is a conformal vector field. Using Lemma 5 , we get $\|A\|^{2}=2 n$. Therefore, $M$ is a Clifford hypersurface (cf. [5]).

The converse is trivial.

Author Contributions: Conceptualization, S.D. and I.A.-D.; methodology, S.D.; software, I.A.-D.; validation, S.D. and I.A.-D.; formal analysis, S.D.; investigation, I.A.-D.; resources, S.D.; data curation, I.A.-D.; writing-original draft preparation, S.D. and I.A.-D.; writing-review and editing, S.D. and I.A.-D.; visualization, I.A.-D.; supervision, S.D. All authors have read and agreed to the published version of the manuscript.

Funding: This research received no external funding. 
Acknowledgments: This work is supported by King Saud University, Deanship of Scientific Research, College of Science Research Center.

Conflicts of Interest: The authors declare no conflict of interest.

\section{References}

1. Simons, J. Minimal varieties in riemannian manifolds. Ann. Math. 1968, 88, 62-105. [CrossRef]

2. Lawson, H.B., Jr. Local rigidity theorems for minimal hypersurfaces. Ann. Math. 1969, 89, $187-197$. [CrossRef]

3. Min, S.H.; Seo, K. A characterization of Clifford hypersurfaces among embedded constant mean curvature hypersurfaces in a unit sphere. Math. Res. Lett. 2017, 24, 503-534. [CrossRef]

4. Perdomo, O. Rigidity of minimal hypersurface of spheres with constant Ricci curvature. Rev. Colomb. Mat. 2004, 38, 73-85.

5. Chern, S.S.; Carmo, M.D.; Kobayashi, S. Minimal submanifolds of a sphere with second fundamental form of constant length. In Functional Analysis and Related Fields; Springer: New York, NY, USA, 1970; pp. 59-75.

6. Lei, L.; Xu, H.; Xu, Z. On Chern's conjecture for minimal hypersurface in spheres. arXiv 2017, arXiv:1712.01175.

7. Perdomo, O. Another proof for the rigidity of Clifford minimal hypersurfaces of $S^{n}$. Mat. Ins. Univ. 2005, 13, 1-6.

8. Sun, H.; Ogiue, K. Minimal hypersurfaces of unit sphere. Tohoku Math. J. 1997, 149, 423-429. [CrossRef]

9. Haizhong, L. A characterization of Clifford minimal hypersurfaces in $S^{3}$. PAMS 1995, 123, 3183-3187. [CrossRef]

10. Montes, R.R.; Verderesi, J.A. Minimal surfaces in $S^{3}$ with constant contact angle. Monatsh. Math. 2009, 157, 379-386. [CrossRef]

11. Montes, R.R.; Verderesi, J.A. Contact angle for immersed surfaces in $S^{2 n+1}$. Diff. Geom. Appl. 2007, 25, 2-100. [CrossRef]

12. Wang, Q. Rigidity of Clifford minimal hypersurfaces. Monatsh. Math. 2003, 140, 163-167. [CrossRef]

13. Blair, D.E. Contact Manifolds in Riemannian Geometry; Lecture Notes in Mathematics; Springer: Berlin, Germany, 1976; Volume 509.

14. Chen, B.-Y. Total Mean Curvature and Submanifolds of Finite Type, Volume 1 of Series in Pure Mathematics; World Scientific Publishing Co.: Singapore, 1984.

15. Yano, K. Integral Formulas in Riemannian Geometry; Marcel Dekker: New York, NY, USA, 1970. 